\title{
Enhanced expression of human cyclin G1 (CCNG1) in tumors, a novel biomarker in development for cancer therapy/gene therapy
}

\author{
Joshua R. Ravicz ${ }^{1,2}$, Cristopher Szeto ${ }^{3}$, Sandeep Reddy ${ }^{4}$, Sant P. Chawla ${ }^{2}$, Michael A. Morse ${ }^{5}$, \\ Erlinda M. Gordon ${ }^{2,6,7}$ \\ ${ }^{1}$ University of Virginia School of Medicine, Charlottesville, VA 22903, ${ }^{2}$ Cancer Center of \\ Southern California/Sarcoma Oncology Center, Santa Monica CA 90403, ${ }^{3}$ NantOmics, Culver \\ City, CA, ${ }^{4}$ NantHealth, Culver City, CA, ${ }^{5}$ Duke University Medical Center, ${ }^{6}$ Delta Next-Gene, \\ LLC, Santa Monica CA, ${ }^{7}$ Aveni Foundation, Santa Monica CA 90405
}

Short Title: $C C N G 1$, a novel biomarker for cancer therapy/gene therapy

Keywords: DeltaRex-G, human cyclin G1, cell cycle control, cancer gene therapy, oncogenic drivers

\author{
Address Correspondence to: \\ Erlinda M. Gordon, MD \\ 2811 Wilshire Blvd., Suite 414 \\ Santa Monica CA 90403 \\ Email: egordon@sarcomaoncology.com \\ Tel: 818-726-3278
}

\begin{abstract}
Background: Metastatic cancer is associated with an invariably fatal outcome. However, DeltaRex-G, a tumor- targeted retrovector encoding a CCNG1 inhibitor gene, has induced long term ( $>10$ years) survival of patients with chemo-resistant metastatic pancreatic adenocarcinoma, MPNST, osteosarcoma, B-cell lymphoma, and breast carcinoma. Objective: To evaluate the level of $C C N G 1$ expression in tumors as a potential biomarker for $C C N G 1$ inhibitor therapy. Methods: $C C N G 1$ RNA expression levels were measured in tumors (TCGA, N=9161), adjacent "tissues" (TCGA, $\mathrm{N}=678)$ and GTEx normal tissues $(\mathrm{N}=7187)$ across 22 organ sites. Differential expression of $C C N G 1$ and Ki-67 in primary $(\mathrm{N}=9161)$ vs metastatic $(\mathrm{N}=393)$ tumors were also compared and particularly in primary $(\mathrm{N}=103)$ vs. metastatic $(\mathrm{N}=367)$ skin cancer (i.e., melanoma). Results: Enhanced $C C N G 1$ RNA and protein expression were noted in tumors compared to normal analogous counterparts, and CCNG1 expression correlated significantly with that of Ki-67. Further, CCNG1 expression tended to be higher than that of Ki-67 in metastatic vs primary tumors, particularly in skin cancer (melanoma). Conclusions: Taken together, these data indicate that (1) $C C N G 1$ expression is frequently enhanced in tumors when compared to their normal analogous counterparts, (2) CCNG1 and Ki-67 expressions are higher in metastatic vs primary tumors, (3) $C C N G 1$ expression is significantly correlated with that of $\mathrm{Ki}-67$, and (4) CCNG1 may be a stronger marker of metastasis than Ki-67. Phase 2 studies are planned to identify patients who are likely to respond favorably to $C C N G 1$ inhibitor therapy by correlating treatment outcome parameters with $C C N G 1$ expression levels in various cancer types.
\end{abstract}




\section{INTRODUCTION}

Cyclins are a family of regulatory proteins that exert control over cell cycle progression through interaction with cyclin-dependent kinases $(\mathrm{CDKs})^{1}$. In particular, the cyclin G1 gene, $C C N G 1$, is known to have a negative effect on stabilization of p53 through Mdm2- and ATMdependent mechanisms, thus assisting in progression through multiple p53-regulated cell cycle checkpoints, namely the G1/S and G2 checkpoints. ${ }^{2-5}$ Cyclin G1 dysregulation is thus believed to have a role in aberrant cell division and tumorigenesis, and overexpression of cyclin G1 has been noted in breast and colorectal cancers, and is important in hepatocellular carcinoma metastasis. ${ }^{6-8}$ As such, $C C N G 1$ has been suggested as a possible target for antineoplastic gene therapy.

DeltaRex-G, previously known as Rexin-G, is an amphotropic MLV-based retroviral vector displaying (1) a Signature (SIG)- binding peptide and (2) encoding a cytocidal CCNG1 inhibitor gene. When administered intravenously, the DeltaRex-G nanoparticles track down and accumulate in the tumor microenvironment (TME) through binding to abnormal SIG proteins exposed during tumor invasion and angiogenesis ${ }^{9-11 . ~ T r e a t m e n t ~ w i t h ~ D e l t a R e x-G ~ h a s ~}$ demonstrated clinical benefit and prolonged survival in metastatic, chemotherapy-resistant sarcoma, pancreatic ductal adenocarcinoma, and ductal carcinoma of the breast ${ }^{9,12,13}$. Nonetheless, due to the nature of its mechanism, DeltaRex-G may be most efficacious in tumors that overexpress $C C N G 1$. Herein, we report augmented $C C N G 1$ expression in many solid tumors and provide the basis for investigating $C C N G 1$ expression levels in tumors to identify patients who are likely to benefit from $C C N G 1$ inhibitor therapy.

\section{METHODS AND MATERIALS}

Archived tumor samples and post-mortem samples are de-identified tissue samples previously used for other histopathologic examination and molecular profiling studies at NantOmics Bioinformatics Department of Nant and at the Cancer Center of Southern California. Therefore, we believe that FDA, IRB and patient informed consent were not needed.

\section{CCNG1 RNA Sequence Analysis}

We obtained CCNG1 and Ki-67 RNA expression levels in tumors (The Cancer Genome Atlas [TCGA], n=9161), tumor-adjacent tissues (TCGA, n=678) and normal tissues (GenotypeTissue Expression Portal [GTEx], $\mathrm{n}=7187$ ) across 22 organ sites. We also evaluated cohort-level differential expression of $C C N G 1$ and Ki-67 in primary $(n=9161)$ vs metastatic $(n=393)$ tumors, as well as in skin cancer specifically ( $n=103$ and $n=367$, respectively). Further, differences in CCNG1 and Ki-67 expression were analyzed in 28 paired primary and metastatic tumor samples (TCGA).

\section{Qualification of IHC assay for Cyclin G1 expression}

An anti-cyclin G1 IgG1 kappa light chain murine monoclonal antibody (Santa Cruz Biotechnology, Santa Cruz, CA, cat\# sc-8016), coupled with MACH4 anti-mouse secondary antibody (BioCare Medical, Pacheco, CA) were used for immunohistochemical analysis. The mouse monoclonal antibody was optimized on cell line tissue microarray and normal tissue microarray samples sectioned at approximately $4 \mu \mathrm{m}$ and adhered to positively charged glass. Multiple antigen retrieval methods and a series of titrations of the cyclin G1 antibody were performed using the tissue microarray slides to determine the optimal antigen retrieval method and antibody concentration. Once the antibody concentration was chosen, accuracy, specificity, sensitivity, and the range of staining intensity was assessed using de-identified samples of 
hepatocellular carcinoma and breast carcinoma samples as positive controls, and normal tissues as negative controls. Additionally, three neoplastic tissues representing various degrees of cyclin G1 expression were chosen for an assessment of reproducibility and precision. Then, immunohistochemical analysis was performed on 27 de-identified formalin-fixed, deparaffinized samples, representing 13 types of cancer and 4 types of normal tissue. The optimized method is as follows: First, the samples were deparaffinized and rehydrated, and endogenous peroxidase activity was blocked. Then, antigen retrieval was performed in a decloaking chamber with TrisEDTA for 20 minutes at $95^{\circ} \mathrm{C}$. The anti-cyclin G1 antibody was applied at a dilution of 1:2000, and incubated for 30 minutes at room temperature, followed by application of the MACH4 mouse secondary antibody for 15 minutes at room temperature. A MACH4 HRP Polymer (BioCare Medical, Pacheco, CA) was then applied as the detection system, and incubated for 30 minutes at room temperature. The resulting product was incubated in chromogen DAB-beta (BioCare Medical, Pacheco, CA) for 10 minutes at room temperature, and was then counterstained with hematoxylin, dehydrated, cleared in xylene, and finally a coverslip was applied. The samples were then analyzed by a board-certified pathologist who scored the staining intensities of the samples on a scale of 0 to $3+$.

Reproducibility was tested in several ways: (1) intra-run reproducibility, with identical slides run in triplicate in one run on one day; (2) inter-run reproducibility with identical slides tested on three separate runs in three separate days; (3) inter-tech reproducibility, with identical slides run by two different technologists in two separate runs; and (4) inter-instrument reproducibility with identical slides run by the same technologist on two different instruments. Concordance factors were calculated based on the pathologist's scoring of the reproducibility slides. As an indirect confirmation of cyclin G1 assay specificity, additional immunohistochemical experiments were performed to compare expression of cyclin G1 to that of cellular proliferation marker Ki-67.

Intra-run, inter-run, inter-tech, and inter-instrument reliability were all deemed as acceptable and CCNGI expression observed by IHC was in line with that predicted in the literature.

\section{RESULTS}

\section{Points to Consider \#1: CCNG1 is overexpressed throughout the TME, including adjacent non-tumorous areas.}

Figure 1 shows the differential expression of CCNG1 (x-axis) vs Ki-67 (y-axis) in tumor (green) and normal (blue and red) settings. There was no significant difference in CCNG1 and Ki-67 expression across organ sites in TCGA tumor and adjacent non-tumor tissue within the TME (TCGA normal). In contrast, as shown in Table 1, CCNG1 expression was significantly enhanced in TCGA tumor compared to normal tissue obtained post-mortem from patients with no tumors (GTEX normal). This is an important observation since proliferative tumor-associated microvasculature (TAM) and tumor-associated fibroblasts (TAFs) would also show enhanced expression of CCNG1 and Ki-67 in the adjacent non-tumor tissue within the TME. 


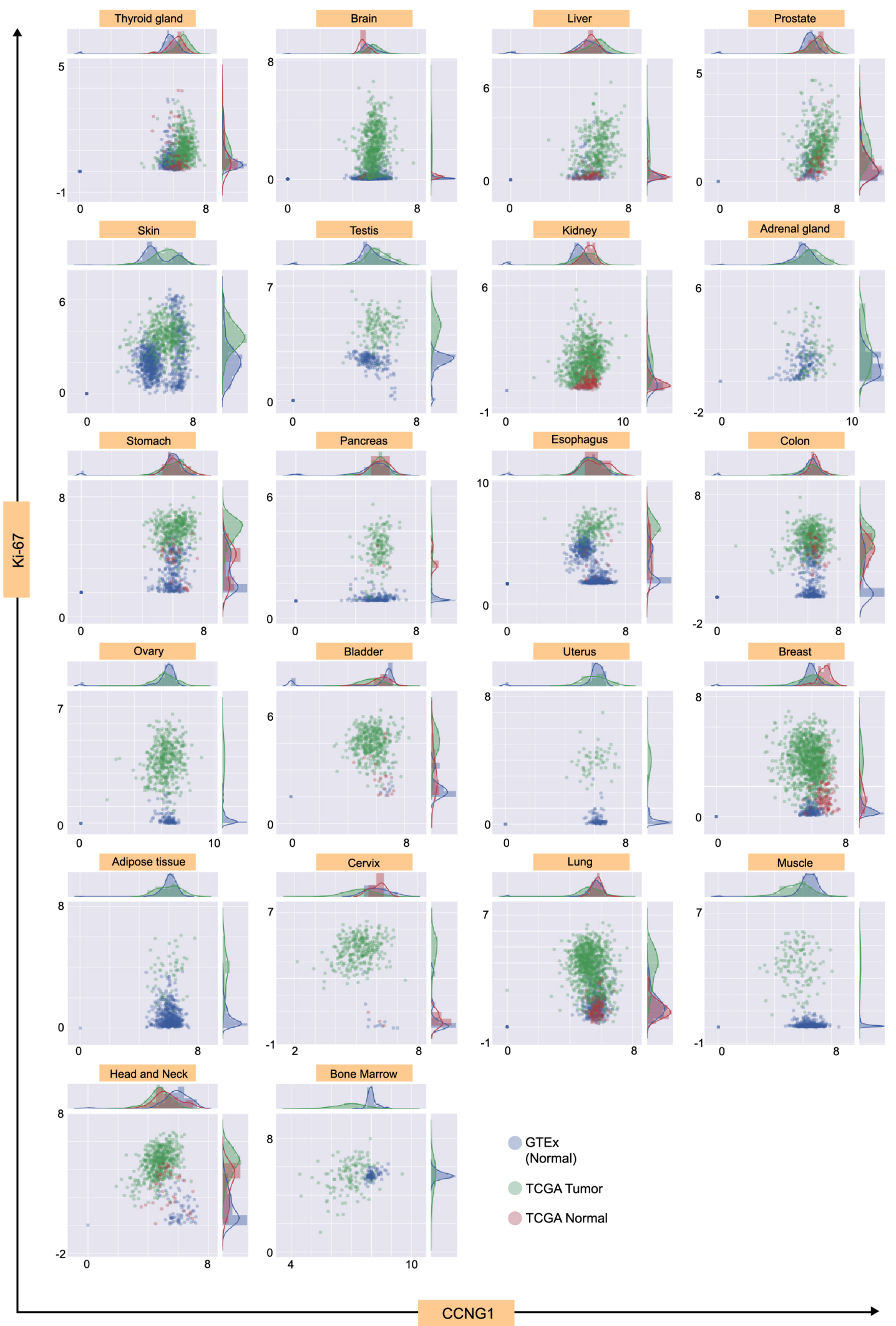

Figure 1 C CNG1 expression across organ sites in normal and tumor tissues, 
contrasted with Ki-67. Scatter plots of CCNG1 (x-axis) vs. Ki-67 (y-axis) for tumor (green) and normal (blue and red) settings. Plots are ordered by differential expression between TCGA tumor and GTEx normal. TCGA normal tissue has altered molecular expression status closer to the TCGA tumor and GTEX normal is distinctly different from both TCGA normal and TGCA tumor.

Table 1. TCGA tumor versus GTEx normal CCNG1 RNA expression, and TCGA tumor versus TCGA tumor-adjacent normal tissue CCNG1 RNA expression

\begin{tabular}{|c|c|c|c|c|}
\hline Tissue type & $\begin{array}{c}\text { TCGA tumor vs. } \\
\text { GTEX t-statistic }\end{array}$ & $\begin{array}{c}\text { TCGA tumor vs. } \\
\text { GTEX p-value }\end{array}$ & $\begin{array}{c}\text { TCGA tumor vs. } \\
\text { TCGA normal t- } \\
\text { statistic }\end{array}$ & $\begin{array}{c}\text { TCGA tumor vs } \\
\text { TCGA normal p- } \\
\text { value }\end{array}$ \\
\hline Thyroid gland & 17.4053 & $1.82761 \mathrm{e}-58$ & 5.77623 & $1.25923 \mathrm{e}-8$ \\
\hline Brain & 10.7032 & $4.58349 \mathrm{e}-26$ & 2.78716 & 0.0054646 \\
\hline Liver & 9.54118 & $5.73639 \mathrm{e}-20$ & 4.66959 & $2.06486 \mathrm{e}-6$ \\
\hline Prostate & 9.42913 & $7.98963 \mathrm{e}-20$ & 1.3379 & 0.181479 \\
\hline Skin & 7.86357 & $7.2756 \mathrm{e}-15$ & NaN & NaN \\
\hline Testis & 6.05271 & $3.58683 \mathrm{e}-9$ & None & None \\
\hline Kidney & 5.98885 & $3.00984 \mathrm{e}-9$ & -2.55981 & 0.0106152 \\
\hline Adrenal gland & 5.37486 & $1.83838 \mathrm{e}-7$ & None & None \\
\hline Stomach & 4.52719 & $6.98267 \mathrm{e}-6$ & -0.679563 & 0.497131 \\
\hline Pancreas & 2.79005 & 0.0055392 & -0.566826 & 0.571535 \\
\hline Esophagus & 1.70762 & 0.088022 & -1.48117 & 0.140167 \\
\hline Colon & 1.53667 & 0.124732 & -2.77403 & 0.00572754 \\
\hline Ovary & 0.466719 & 0.640887 & None & None \\
\hline Bladder & 0.0242461 & 0.980668 & -3.07999 & 0.00220318 \\
\hline Uterus & 0.00963925 & 0.992322 & None & None \\
\hline Breast & -0.0198831 & 0.98414 & -12.4645 & $1.10424 \mathrm{e}-33$ \\
\hline Cervix & -4.08242 & $5.655564 \mathrm{e}-5$ & -2.06655 & 0.039615 \\
\hline Lung & -5.34723 & $1.03968 \mathrm{e}-7$ & -6.02 & $2.34085 \mathrm{e}-9$ \\
\hline $\begin{array}{c}\text { Head and neck } \\
\text { region }\end{array}$ & -11.8523 & $3.22551 \mathrm{e}-29$ & -4.97295 & $8.76887 \mathrm{e}-7$ \\
\hline Soft tissue, bone & -26.7181 & $1.5217 \mathrm{e}-87$ & -1.18341 & 0.237721 \\
\hline
\end{tabular}

\section{Points to Consider \#2: CCNG1 expression is higher than that of Ki-67 in metastatic skin cancers (i.e., melanoma).}

As shown in Figure 2 left panel, $C C N G 1$ and Ki-67 both showed enhanced expression in metastatic settings (orange) compared to primary tumors (green), but CCNG1 outperformed Ki67 specifically in metastatic skin cancer (melanoma; right panel). Further, CCNG1 expression was relatively higher in many metastatic samples than that of Ki-67 and did not track with Ki-67 (Figure 3). These data indicate that CCNG1 gene expression may be a better indicator of metastasis than primary tumors. 

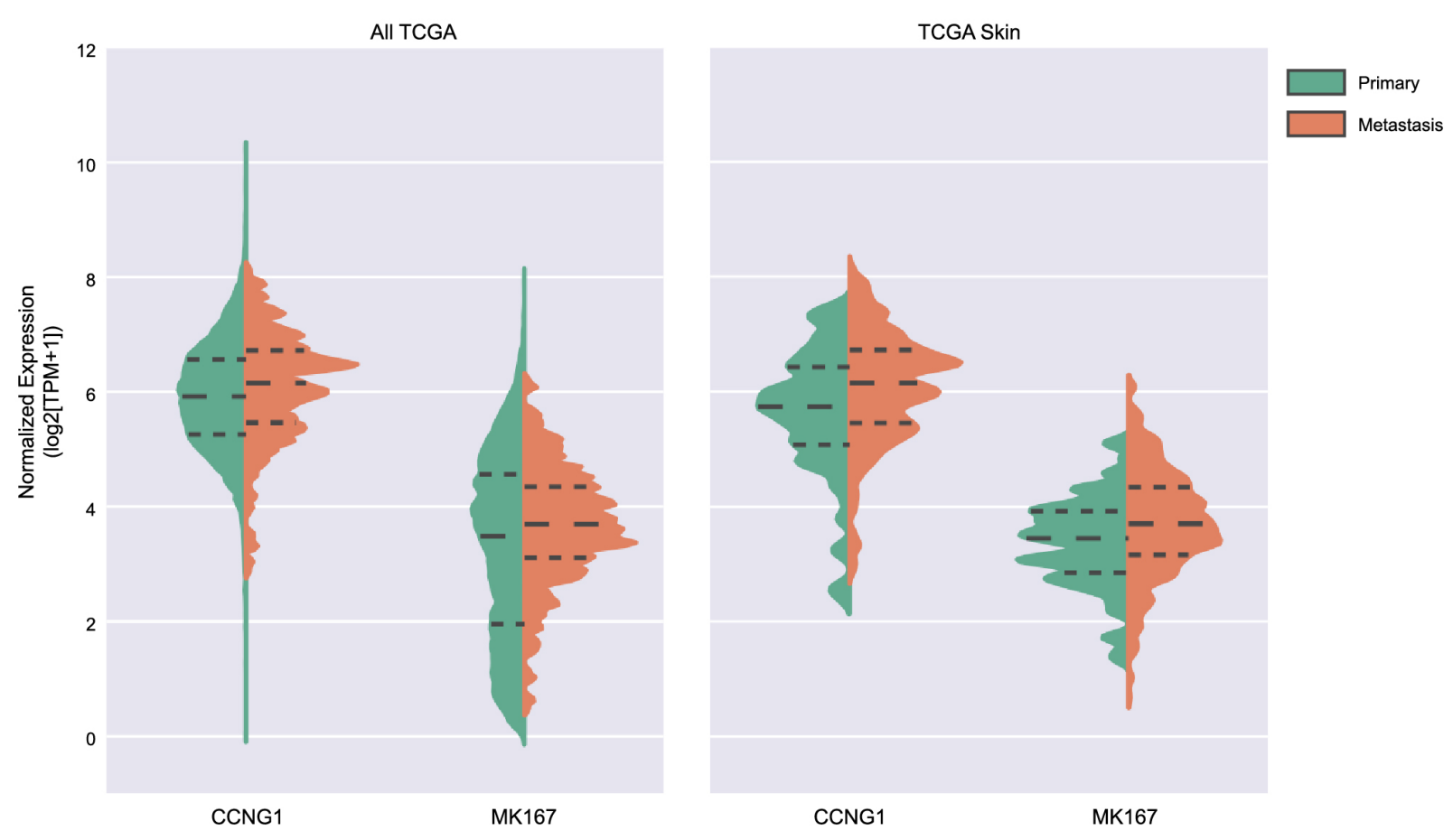

Figure 2 CCNG1 and Ki-67 both have increased expression in the metastatic setting, CCNG1 outperforms Ki-67 in skin specifically. Violin plots showing distribution of normalized CCNG1 and Ki67 expression in primary (green) and metastatic (orange) settings across all TCGA (left) and the skin cancer subset (right).

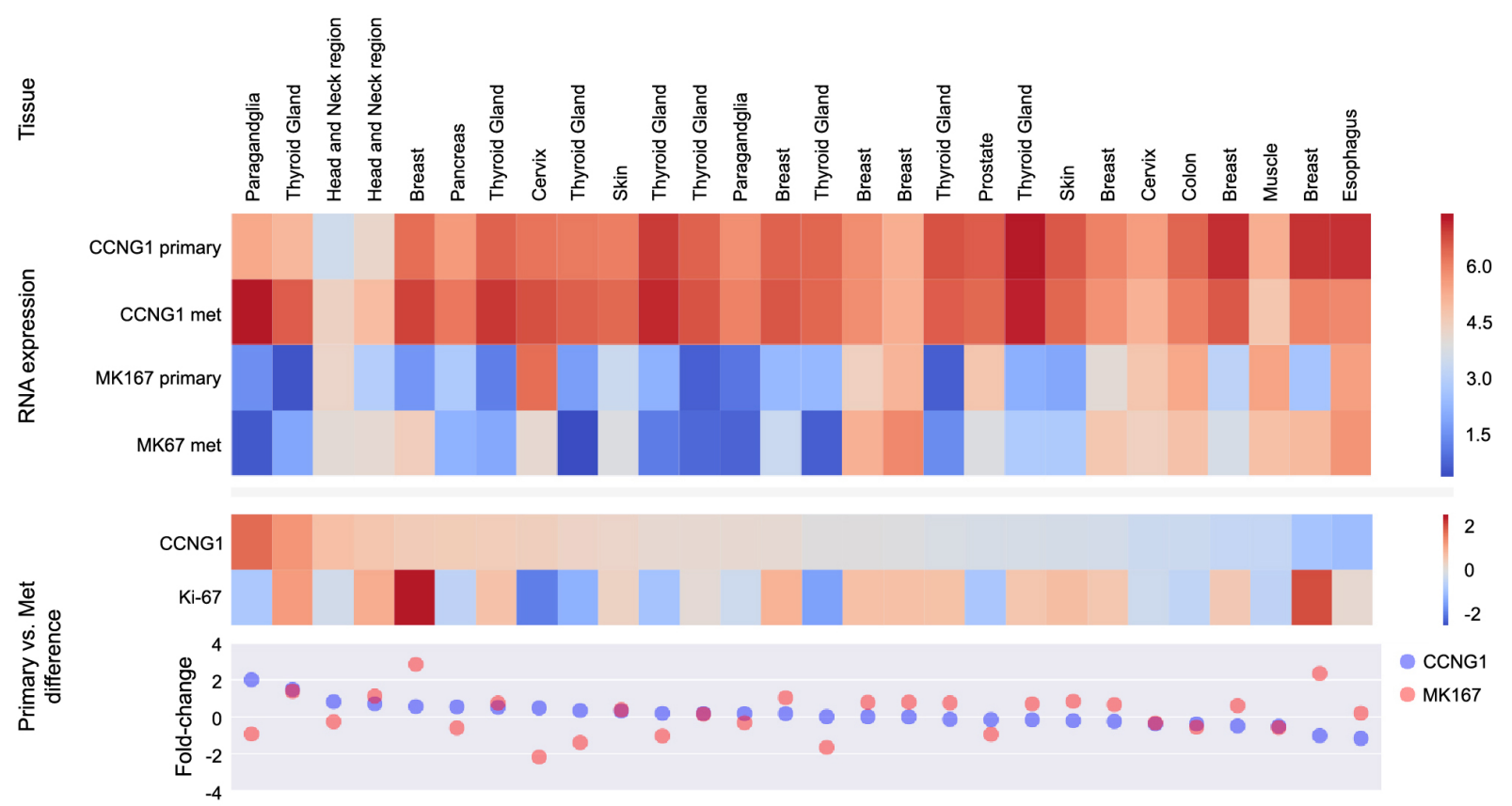

Figure 3 CCNG1 may be an independent metastasis marker from Ki-67. Expression (top) and fold-changes (bottom) of CCNG1 and Ki-67 from 28 samples with both 
primary and met samples in TCGA. CCNG1 expression is relatively higher in many metastatic samples than that of Ki-67 and does not track with Ki-67.

\section{Points to Consider \#3: CCNG1 expression is enhanced in specific tumors by RNA sequence analysis.}

As shown in Table 1, in 11 of 22 measured samples, CCNG1 RNA expression was significantly overexpressed in TCGA tumor samples compared to GTEx normal tissues, including thyroid, brain, liver, prostate, skin, testis, kidney, adrenal, stomach, pancreas and esophageal cancers. In 4 of the 22 samples, $C C N G 1$ RNA was under-expressed in the tumor samples, indicating that these tumors were in dormant or inactive state.

Points to Consider \#4: CCNG1 nuclear protein expression is enhanced in most tumors examined by IHC.

Furthermore, IHC staining revealed significant cyclin G1 overexpression in almost all tumor samples and cancer cell lines (Table 2). For example, Figure 4 shows 80\% nuclear $C C N G 1+$ and nuclear Ki-67+ tumor cells in mesenchymal chondrosarcoma metastatic to brain.
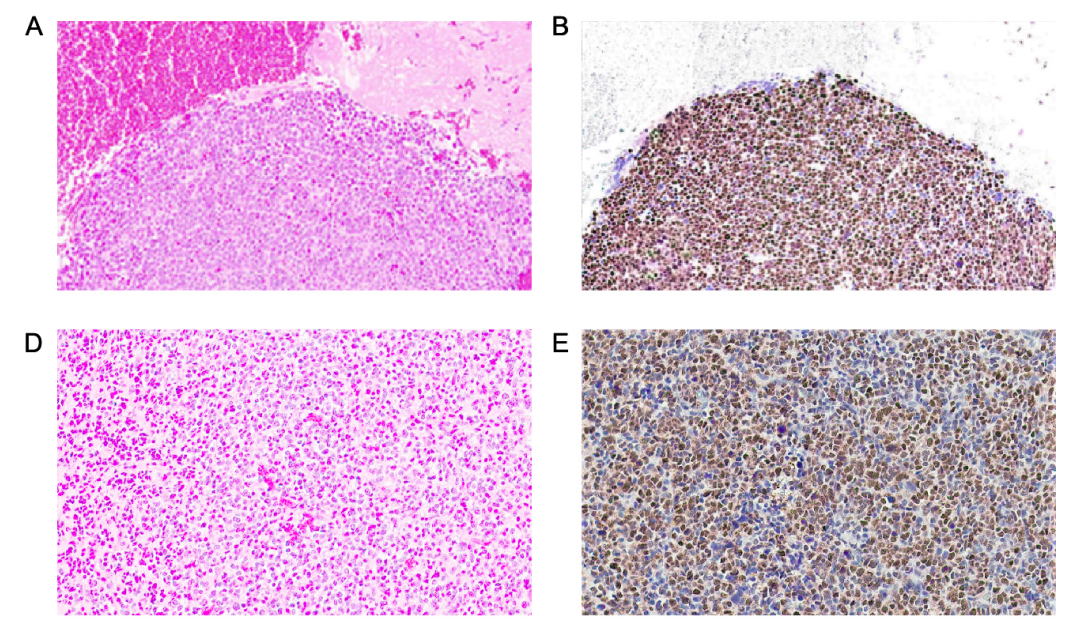
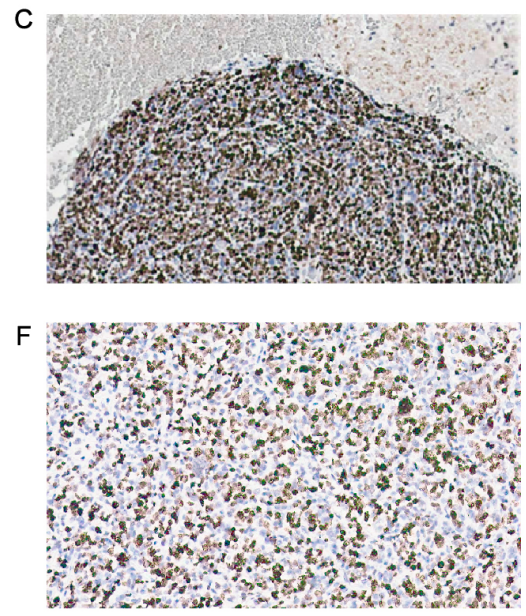

Figure 4 Photomicrograph sections showing enhanced expression of CCNG1 nuclear protein (IHC staining) in mesenchymal chondrosarcoma metastatic to brain. From left to right, A\&D (4X and 20X mag): H\&E stain; B\&E (4X and 20X mag): $80 \%$ CCNG1+ cancer cells; C\&F (4X and 20X mag): $80 \%$ Ki-67+ cancer cells in biopsied specimen of chondrosarcoma metastatic to the brain.

Table 2. Summary of cyclin G1 expression, as ascertained by IHC, for normal vs cancerous tissues.

\begin{tabular}{|c|c|c|c|}
\hline Normal tissue & $\begin{array}{c}C C N G 1 \text { nuclear } \\
\text { staining percentage }\end{array}$ & Cancer cell line (tumor) & $\begin{array}{c}C C N G 1 \text { nuclear } \\
\text { staining percentage }\end{array}$ \\
\hline Breast & $5 \%$ & Breast ILC (tumor) & $20 \%$ \\
\hline & & Breast IDC (tumor) & $35 \%$ \\
\hline
\end{tabular}




\begin{tabular}{|c|c|c|c|}
\hline & & Breast IDC (tumor) & $95 \%$ \\
\hline & & Breast IC (tumor) & $60 \%$ \\
\hline & & $\begin{array}{c}\text { SK-BR3 breast } \\
\text { carcinoma }\end{array}$ & $95 \%$ \\
\hline \multirow[t]{4}{*}{ Liver } & $1 \%$ & HCC (tumor) & $90 \%$ \\
\hline & & HCC (tumor) & $40 \%$ \\
\hline & & HCC (tumor) & $5 \%$ \\
\hline & & HCC (tumor) & $0 \%$ \\
\hline Lung & $0 \%$ & HCC-78 NSCLC & $90 \%$ \\
\hline Brain, cerebellum & $0 \%$ & T98G glioblastoma & $100 \%$ \\
\hline Tongue & $0 \%$ & Sarcoma NOS (tumor) & $70 \%$ \\
\hline \multirow[t]{5}{*}{ Skeletal muscle } & $0 \%$ & $\begin{array}{c}\text { Leiomyosarcoma } \\
\text { (tumor) }\end{array}$ & $30 \%$ \\
\hline & & Angiosarcoma (tumor) & $90 \%$ \\
\hline & & Liposarcoma (tumor) & $40 \%$ \\
\hline & & $\begin{array}{l}\text { Chondrosarcoma } \\
\text { (tumor) AD primary } \\
\text { tumor } 2015\end{array}$ & $0 \%$ \\
\hline & & $\begin{array}{c}\text { Chondrosarcoma } \\
\text { (tumor) AD metastatic } \\
\text { to brain } 2017\end{array}$ & $80 \%$ \\
\hline \multirow[t]{2}{*}{ Colon epithelium } & $30 \%$ & $\begin{array}{l}\text { Colorectal carcinoma } \\
\text { (tumor) ST metastatic } \\
\text { to liver } 2016\end{array}$ & $90 \%$ \\
\hline & & $\begin{array}{c}\text { Colorectal carcinoma } \\
\text { (tumor) ST metastatic } \\
\text { to liver } 2017\end{array}$ & $20 \%$ \\
\hline None & & $\begin{array}{l}\text { HDLM2 Hodgkin } \\
\text { lymphoma }\end{array}$ & $95 \%$ \\
\hline None & & $\begin{array}{c}\text { Jurkat T lymphoblastic } \\
\text { leukemia }\end{array}$ & $95 \%$ \\
\hline None & & Karpas ALL & $100 \%$ \\
\hline None & & $\begin{array}{l}\text { Jeko-1 mantle cell } \\
\text { lymphoma }\end{array}$ & $95 \%$ \\
\hline
\end{tabular}

\section{DISCUSSION}

As cancer treatment continues to trend toward more highly directed molecular therapies, efforts have been made to determine how to best assess which patients may benefit from specific targeted therapies. Cyclin G1 dysregulation is an important down regulator of p53 activity, allowing for disinhibited cell cycle progression. ${ }^{14-16}$ In the present study, we found that cyclin G1 is overexpressed in many tumor samples, and that in metastatic tumors, cyclin G1 expression is higher than that of Ki-67. Currently, DeltaRex-G, a retrovector encoding a CCNG1 inhibitor gene is in development as therapy for various cancer types, and should be included as part of molecular profiling of tumors at diagnosis and in post-treatment tumor specimens 
DeltaRex-G is the first, and so far, only, tumor-targeted retrovector encoding a CCNG1 inhibitor gene. ${ }^{10,11,13,17}$ DeltaRex-G has been found to be of clinical benefit in an array of metastatic cancers, ${ }^{9,18}$ and the ability to identify patients with specific overexpression of CCNG1 can help inform clinicians as to which patients may benefit the most from this gene therapy. Additionally, our finding that CCNG1 expression has varied over time in some tumors and suggests that genomic data from multiple biopsies or from circulating cell-free tumor DNA over time may be most informative relating to the optimal timing of DeltaRex-G and other future CCNG1 inhibitor therapies.

Our findings that in some tumors, cyclin G1 is overexpressed in metastatic sites when compared to primary tumors piques our interest into the role of cyclin G1 and CCNG1 inhibitors in the treatment of metastatic disease. One important pathway in the epithelial-mesenchymal transition (EMT), a crucial process in tumor metastasis and dissemination, is downregulation of E-cadherin. ${ }^{19,20}$ Physiologically, GSK-3 $\beta$ phosphorylates Snail (the most important transcription factor in negative regulation of E-cadherin), causing its degradation, and thus disallowing it from downregulating E-cadherin. ${ }^{21,22}$ Cyclin G1 is thought to incite EMT by activating PI3K/Akt signaling to downregulate GSK-3 $\beta$, eventually leading to loss of E-cadherin. ${ }^{8}$ Conceptually, DeltaRex-G would inhibit EMT and downregulate the PI3K/Akt signaling pathway as well. The data showing overexpression of cyclin G1 at primary and metastatic tumors solidifies the case for $C C N G 1$ inhibition for cancer therapy/gene therapy and the results of an advanced Phase $1 / 2$ study of DeltaRex-G in the metastatic pancreatic adenocarcinoma have been successful in gaining orphan drug and fast track designation from the USFDA ${ }^{23}$.

We also found cyclin G1 to be overexpressed not just in tumors, but within the TME as well. The angiogenic switch is the term used to describe the time in tumor progression when proangiogenic factors begin to predominate over anti-angiogenic factors, leading to vascular proliferation in the TME, allowing the tumor to receive nutrients required to progress and to maintain its high energy demand. ${ }^{24,25}$ Additionally, TME vasculature encourages evasion of antitumor immune surveillance, in part by impeding lymphocyte-endothelial cell interaction and upregulating immunosuppressive leukocytes. ${ }^{25}$ The specific cytocidal activity of DeltaRex-G in TAM and TAFs suggests that DeltaRex-G breaks anergy by enhancing immune cell trafficking in the TME. ${ }^{11}$ Future studies should investigate possible synergy between DeltaRex-G administered with other anti-angiogenic therapies like $n a b$-sirolimus, an mTOR inhibitor, and immune checkpoint inhibitors such as nivolumab and ipilimumab. ${ }^{26}$ Phase 2 studies are planned to identify patients who are likely to respond favorably and/or benefit most to DeltaRex-G therapy by correlating $C C N G 1$ expression levels and treatment outcome parameters in cancer patients treated with DeltaRex-G.

\section{Acknowledgements:}

The authors are grateful to Heather Gordon for technical support and graphic illustrations, and J Isaacs Charitable Trust UK, Thomas Makin Family, James B. Finn Memorial, ArtistsforAveni, Capital Group, Trader Joes, Holmes Family Trust, Leah Chelsky Family Fund, Lance S. Ostendorf, Adolf Weinberger Foundation, Martin R. Berwitt, Lawrence Yaeger Memorial Fund, Frederick L. Hall, PhD, and Don A. Brigham for their generous donations. 


\section{REFERENCES}

1. Malumbres, M. (2014). Cyclin-dependent kinases. Genome Biology 15, 122.

2. Giono, L.E., and Manfredi, J.J. (2006). The p53 tumor suppressor participates in multiple cell cycle checkpoints. Journal of Cellular Physiology 209, 13-20.

3. Ohtsuka, T., Ryu, H., Minamishima, Y.A., Ryo, A., and Lee, S.W. (2003). Modulation of p53 and p73 levels by cyclin G: implication of a negative feedback regulation. Oncogene 22, 16781687.

4. Ohtsuka, T., Jensen, M.R., Kim, H.G., Kim, K., and Lee, S.W. (2004). The negative role of cyclin $\mathrm{G}$ in ATM-dependent p53 activation. Oncogene 23, 5405-5408.

5. Okamoto, K., and Prives, C. (1999). A role of cyclin G in the process of apoptosis. Oncogene $18,4606-4615$.

6. Perez, R., Wu, N., Klipfel, A.A., and Beart, R.W. (2003). A better cell cycle target for gene therapy of colorectal cancer: cyclin G. J. Gastrointest. Surg. 7, 884-889.

7. Reimer, C.L., Borras, A.M., Kurdistani, S.K., Garreau, J.R., Chung, M., Aaronson, S.A., and Lee, S.W. (1999). Altered regulation of cyclin $\mathrm{G}$ in human breast cancer and its specific localization at replication foci in response to DNA damage in p53+/+ cells. J. Biol. Chem. 274, 11022-11029.

8. Wen, W., Ding, J., Sun, W., Fu, J., Chen, Y., Wu, K., Ning, B., Han, T., Huang, L., Chen, C., et al. (2012). Cyclin G1-mediated epithelial-mesenchymal transition via phosphoinositide 3kinase/Akt signaling facilitates liver cancer progression. Hepatology 55, 1787-1798.

9. Chawla, S.P., Bruckner, H., Morse, M.A., Assudani, N., Hall, F.L., and Gordon, E.M. (2018). A Phase I-II Study Using Rexin-G Tumor-Targeted Retrovector Encoding a Dominant-Negative Cyclin G1 Inhibitor for Advanced Pancreatic Cancer. Mol Ther Oncolytics 12, 56-67.

10. Gordon, E.M., and Hall, F.L. (2010a). Rexin-G, a targeted genetic medicine for cancer. Expert Opin Biol Ther 10, 819-832.

11. Hall, F.L., Liu, L., Zhu, N.L., Stapfer, M., Anderson, W.F., Beart, R.W., and Gordon, E.M. (2000). Molecular engineering of matrix-targeted retroviral vectors incorporating a surveillance function inherent in von Willebrand factor. Hum. Gene Ther. 11, 983-993.

12. Gordon, E.M., and Hall, F.L. (2010b). Noteworthy clinical case studies in cancer gene therapy: Tumor-targeted Rexin-G advances as an efficacious anti-cancer agent. Int J Oncol. 36, 1341-1353.

13. Gordon, E.M., Lopez, F.F., Cornelio, G.H., Lorenzo, C.C., Levy, J.P., Reed, R.A., Liu, L., Bruckner, H.W., and Hall, F.L. (2006). Pathotropic nanoparticles for cancer gene therapy Rexin$\mathrm{G}^{\mathrm{TM}} \mathrm{IV}$ : Three-year clinical experience. Int J Oncol. 29, 1053-1064.

14. Gordon, E.M., Ravicz, J.R., Liu, S., Chawla, S.P., and Hall, F.L. (2018). Cell cycle checkpoint control: The cyclin G1/Mdm2/p53 axis emerges as a strategic target for broadspectrum cancer gene therapy - A review of molecular mechanisms for oncologists. Mol Clin Oncol 9, 115-134.

15. Jensen, M.R., Factor, V.M., Fantozzi, A., Helin, K., Huh, C.-G., and Thorgeirsson, S.S. (2003). Reduced hepatic tumor incidence in cyclin G1-deficient mice. Hepatology 37, 862-870. 16. Okamoto, K., Li, H., Jensen, M.R., Zhang, T., Taya, Y., Thorgeirsson, S.S., and Prives, C. (2002). Cyclin G recruits PP2A to dephosphorylate Mdm2. Mol. Cell 9, 761-771.

17. Gordon, E.M., Levy, J.P., Reed, R.A., Petchpud, W.N., Liu, L., Wendler, C.B., and Hall, F.L. (2008). Targeting metastatic cancer from the inside: a new generation of targeted gene delivery vectors enables personalized cancer vaccination in situ. Int J Oncol. 33, 665-675. 
18. Kim, S., Federman, N., Gordon, E.M., Hall, F.L., and Chawla, S.P. (2017). Rexin-G®, a tumor-targeted retrovector for malignant peripheral nerve sheath tumor: A case report. Mol Clin Oncol 6,861-865.

19. Gheldof, A., and Berx, G. (2013). Cadherins and epithelial-to-mesenchymal transition. Prog Mol Biol Transl Sci 116, 317-336.

20. Thiery, J.P. (2002). Epithelial-mesenchymal transitions in tumour progression. Nature Reviews Cancer 2, 442-454.

21. Cano, A., Pérez-Moreno, M.A., Rodrigo, I., Locascio, A., Blanco, M.J., del Barrio, M.G., Portillo, F., and Nieto, M.A. (2000). The transcription factor Snail controls epithelialmesenchymal transitions by repressing E-cadherin expression. Nature Cell Biology 2, 76-83. 22. Zhou, B.P., Deng, J., Xia, W., Xu, J., Li, Y.M., Gunduz, M., and Hung, M.-C. (2004). Dual regulation of Snail by GSK-3beta-mediated phosphorylation in control of epithelialmesenchymal transition. Nat. Cell Biol. 6, 931-940.

23. Chawla, S.P., Chua, V.S., Fernandez, L., Quon, D., Blackwelder, W.C., Gordon, E.M., and Hall, F.L. (2010). Advanced Phase I/II Studies of Targeted Gene Delivery In Vivo: Intravenous Rexin-G for Gemcitabine-resistant Metastatic Pancreatic Cancer. Mol Ther 18, 435-441.

24. Baeriswyl, V., and Christofori, G. (2009). The angiogenic switch in carcinogenesis. Seminars in Cancer Biology 19, 329-337.

25. Schaaf, M.B., Garg, A.D., and Agostinis, P. (2018). Defining the role of the tumor vasculature in antitumor immunity and immunotherapy. Cell Death Dis 9, 115.

26. Al-Shihabi, A., Chawla, S.P., Hall, F.L., and Gordon, E.M. (2018). Exploiting Oncogenic Drivers along the CCNG1 Pathway for Cancer Therapy and Gene Therapy. Molecular Therapy Oncolytics $11,122-126$. 